\title{
Magnetoabsorption in InSb quantum-well heterostructures
}

\author{
J. M. S. Orr, ${ }^{*}$ K.-C. Chuang, and R. J. Nicholas \\ Clarendon Laboratory, University of Oxford, Parks Road, Oxford OX1 3PU, United Kingdom
}

L. Buckle, M. T. Emeny, and P. D. Buckle

QinetiQ, Malvern WR13 3PS, United Kingdom

(Received 31 March 2009; published 2 June 2009)

\begin{abstract}
Spin-resolved cyclotron resonance is observed in InSb/AlInSb quantum-well heterostructures. Spin-resolved transitions are identified for fields greater than $2.2 \mathrm{~T}$ and confirmed by $\mathbf{k} \cdot \mathbf{p}$ modeling. The cyclotron effective mass is calculated for a range of fields up to $11.5 \mathrm{~T}$ and is indicative of the strong nonparabolicity in this material. Resonant coupling is observed between the ground and first occupied subbands for normal-incidence excitation. This is explained in the context of conduction band-valance band mixing due to the narrow bandgap. Further resonant coupling is observed via interaction with a hybrid intersubband plasmon-LO phonon mode. This allows a quantitative estimation of the subband spacing and of the electron-phonon interactions in the quantum well.
\end{abstract}

DOI: 10.1103/PhysRevB.79.235302

PACS number(s): 78.67.De, 78.66.Fd, 78.30.Fs, 71.38.-k

\section{INTRODUCTION}

Recent interest in InSb-based heterostructures has been driven by the many advantageous material properties available for device applications, in particular its narrow bandgap, low electron effective mass, strong spin-orbit coupling, large Landé $g$ factor, and high electron mobility at room temperature. It has been demonstrated that these characteristics lend themselves to applications such as high electron mobility transistors, ${ }^{1}$ ballistic mesoscopic devices, ${ }^{2}$ magnetic sensors, ${ }^{3}$ and spintronics, where the spin manipulation and control are facilitated by the exceptionally large $g$ factor and therefore also by significant Rashba splitting. ${ }^{4,5}$

In this paper we perform magnetoabsorption measurements on InSb/AlInSb quantum-well heterostructures and identify spin-resolved cyclotron resonance $(\mathrm{CR})$ transitions and Landau level coupling phenomena. This enables the study of the characteristically low effective mass in this material system. In these experiments, the orientation of the sample is defined so that the field, $B$, and the incident wave vector of the signal are collinear and make an angle, $\theta$, with the sample growth direction, $z$. When $\theta=0$ the orientation is referred to as the perpendicular Faraday (PF) configuration, otherwise it is referred to as the tilted Faraday (TF) configuration.

The electronic states in the InSb two-dimensional electron gas (2DEG) are quantized in the $z$ direction into subbands labeled as $E_{i}$, where $i$ is an integer ( $i=1,2$ in these samples). Within each subband the electronic states are further quantized into equally spaced Landau levels (indexed $n=0$ to $\infty$ ) in the $(x, y)$ plane by the application of a magnetic field. Each of these levels is further split by the Zeeman effect according to spin quantum number, with an energy shift $\pm g^{*} \mu_{B} B / 2$, where $g^{*}$ is the effective $g$ factor and $\mu_{B}$ is the Bohr magneton. Cyclotron resonance absorption takes place via spin-conserving transitions across adjacent Landau levels $(\Delta n= \pm 1, \Delta s=0)$. When the energy of the incident light is resonant with the energy gap between the Zeeman-split Landau levels, cyclotron resonance absorption takes place leading to a direct evaluation of the cyclotron mass, $m_{\mathrm{CR}}^{*}$.
Such a situation is shown schematically in Fig. 1, where the Landau level energies of the two subbands are depicted as a function of magnetic field, the dashed (solid) lines represent the spin-up (down) states, and examples of allowed transitions are shown as solid arrows. According to the simple theory when the sample is in the PF configuration there is no interaction between the subband $(z)$ and Landau level $(x, y)$ quantizations; ${ }^{6}$ however if the sample is tilted (TF configuration) the introduction of a small field component parallel to the 2DEG allows the Landau level wave functions of different subbands to mix and results in an anticrossing behavior between Landau levels of the same spin, as shown in Fig. 1 at field $B_{\text {coupling. }}$. Experimentally, this effect is manifested as a broadening or splitting of the cyclotron resonance lines at an energy corresponding to the subband separation,

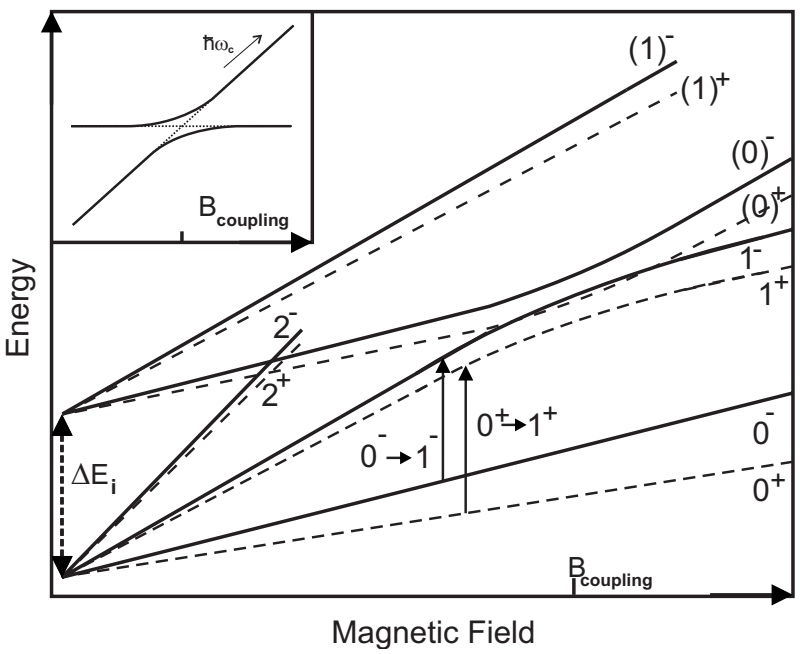

FIG. 1. Schematic of energy-level quantization in a twosubband system $(i=1,2)$ as a function of magnetic field, showing the first three Landau levels, $n=0-2$. Each Landau level is split into spin-up (down) states, dashed (solid) lines. Where Landau levels of the same spin intersect an anticrossing takes place. Inset: cyclotron resonance energy as it passes through the RSLC, noting that the resonance splits into two branches. 
$E_{10} \cdot{ }^{7}$ This is shown schematically in the inset of Fig. 1. An analytical approach has predicted that the upper branch pins to the $E_{10}$ energy level, while the lower branch pins to $E_{10} \cos \theta$, so that the splitting is increased for large tilt angles. ${ }^{6}$ This behavior is known as resonant subband Landau level coupling (RSLC) and is a convenient way of determining the subband energy spacing in a 2 DEG. ${ }^{8}$

The InSb/ $\mathrm{Al}_{x} \mathrm{In}_{1-x} \mathrm{Sb}$ heterostructures reported here were grown by solid source molecular beam epitaxy (MBE) on semi-insulating GaAs substrates. In growth order, the material consists of a strain accommodation system followed by a $3 \mu \mathrm{m} \mathrm{Al} \mathrm{Al}_{0.10} \mathrm{In}_{0.90} \mathrm{Sb}$ strain-relaxed lower barrier layer, a $30 \mathrm{~nm}$ InSb quantum well, and a $50 \mathrm{~nm}$ upper barrier layer of $\mathrm{Al}_{0.15} \mathrm{In}_{0.85} \mathrm{Sb}$. The quantum well is remotely doped by a Te delta-doping layer within the upper barrier, this is separated from the quantum well by a $25 \mathrm{~nm}$ spacer. Finally the sample substrates were polished at small angles $\left(2^{\circ}\right)$ to reduce interference effects in the cyclotron resonance measurements. Schrodinger-Poisson modeling of these structures has been performed and indicates two occupied subbands at liquid-helium temperatures. ${ }^{9}$ Several samples were measured with carrier densities in the range $2.2 \times 10^{11}<n_{s}<3.9$ $\times 10^{11} \mathrm{~cm}^{-2}$ at $4.2 \mathrm{~K}$. All samples gave comparable results and for clarity the following data are presented for a single sample $\left(3.9 \times 10^{11} \mathrm{~cm}^{-2}\right)$. Transport measurements and further growth details for this materials system are reported elsewhere. ${ }^{9,10}$

Absorption spectra were obtained using a Bruker IFS113 Fourier transform (FTIR) spectrometer with a broadband midinfrared source (globar). The signal was guided to the sample via a highly polished light pipe and focusing cone to ensure good illumination and alignment. The samples were mounted within a superconducting magnet and held at $4.2 \mathrm{~K}$, either in PF or TF configuration (with $\theta=6^{\circ}$ ). The transmitted portion of the signal was measured by a silicon bolometer mounted behind the sample, and the absorption data presented were acquired by dividing a zero-field reference by the raw spectra at a given field in order to eliminate any instrument-dependent features.

\section{SPIN-RESOLVED CYCLOTRON RESONANCE}

The ability to observe spin-resolved cyclotron resonance transitions does not in fact depend directly on the magnitude of the Zeeman splitting but on the magnitude of the difference in Zeeman splitting between adjacent Landau levels, as a result this effect is sometimes referred to as the $\Delta g$ splitting. ${ }^{11}$ In InSb the narrow bandgap results in strong nonparabolicity effects, with the result that the $g$ factor exhibits a strong dependence on energy (and magnetic field). This makes InSb an ideal material in which to observe spinresolved cyclotron resonance. ${ }^{4}$ The mechanism that introduces spin-dependence in the cyclotron resonance is illustrated in Fig. 1 where the CR transition labeled as $0^{+} \rightarrow 1^{+}$ has a different energy to the transition $0^{-} \rightarrow 1^{-}$, as a consequence of the difference in $g$ factor between the two Landau levels.

Figure 2 shows the energy positions of the cyclotron resonance transitions measured in the PF configuration between

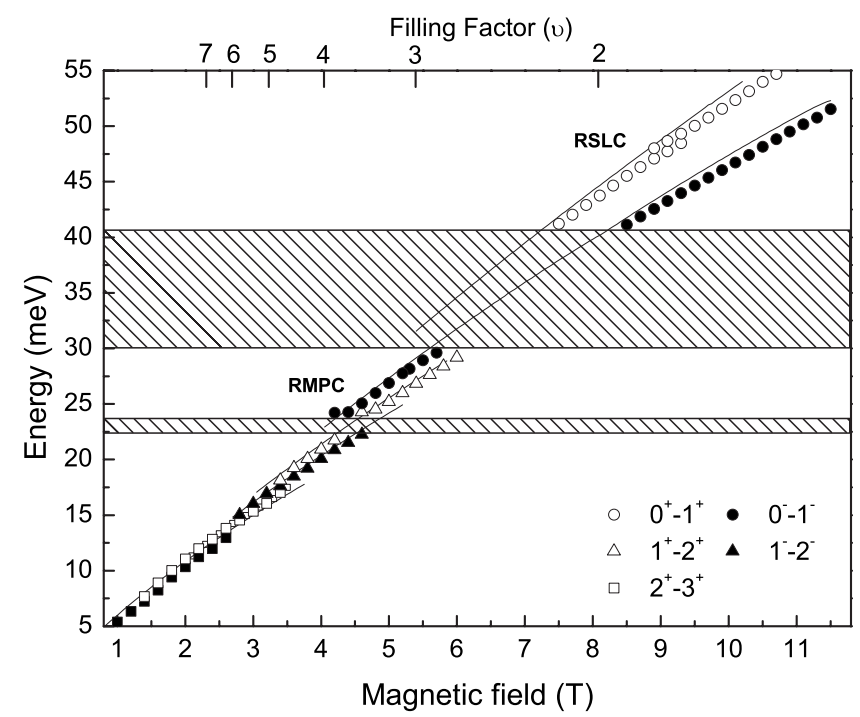

FIG. 2. Cyclotron resonance energy positions as a function of magnetic field in the PF configuration. Hatched areas cover regions of the spectra where data are unreliable due to coincidence with the substrate restrahlen band and the quantum-well TO phonon absorption. Transitions are labeled according to Fig. 1, and resonant coupling behavior is clearly observed between 3.5 and $5.5 \mathrm{~T}$ and between 8.5 and $9.5 \mathrm{~T}$. The calculated filling factors $\nu$ are given along the top axis. Transition energies calculated from $\mathbf{k} \cdot \mathbf{p}$ modeling are shown as solid lines.

1 and $11.5 \mathrm{~T}$ for the InSb heterostructure sample. The Landau level filling factors for this sample were calculated as $\nu=h n_{s} / e B$, where $n_{s}$ is the quantum-well sheet carrier density, and are shown along the top axis. An eight-band $\mathbf{k} \cdot \mathbf{p}$ calculation was performed for this heterostructure and the predicted CR transition energies (solid lines) were derived from the calculated spin-split Landau levels. Two hatched sections in the data are obscured by enhanced absorption; the GaAs substrate restrahlen band (30-41 meV), and a region around the InSb TO phonon energy (22.4-23.7 meV) due to absorption in the AlInSb buffer. Poor signal-to-noise ratio in these regions makes interpretation of any resonance features difficult and unreliable. Resonant coupling behavior can be seen between 8.5 and $9.5 \mathrm{~T}$ and between 3.5 and $5.5 \mathrm{~T}$, and is identified, respectively, as RSLC and resonant magnetopolaron coupling (RMPC). These features are analyzed in greater detail in Secs. III and IV.

The measured CR transitions are in good agreement with those predicted by the $\mathbf{k} \cdot \mathbf{p}$ calculation which helps one to establish the identity of each resonance. Furthermore, each transition is identified by the range of magnetic field over which it is allowed by level occupancy, for example, the $0^{+} \rightarrow 1^{+}$transition should occur for $\nu<3(B>5.4 \mathrm{~T})$, while the $0^{-} \rightarrow 1^{-}$transition should occur in the range $4>\nu>1$ $(4.0<B<16.2 \mathrm{~T})$, and the $1^{+} \rightarrow 2^{+}$transition should occur in the range $5>\nu>2(3.2<B<8.1 \mathrm{~T})$, and so on. With reference to the filling factors presented in Fig. 2, it is clear that the observed transitions comply very closely with the expected ranges, allowing the unambiguous identification of each CR transition.

Having thus identified the CR transitions, it is possible to distinguish the onset of spin-resolved CR from as low as 2.2 


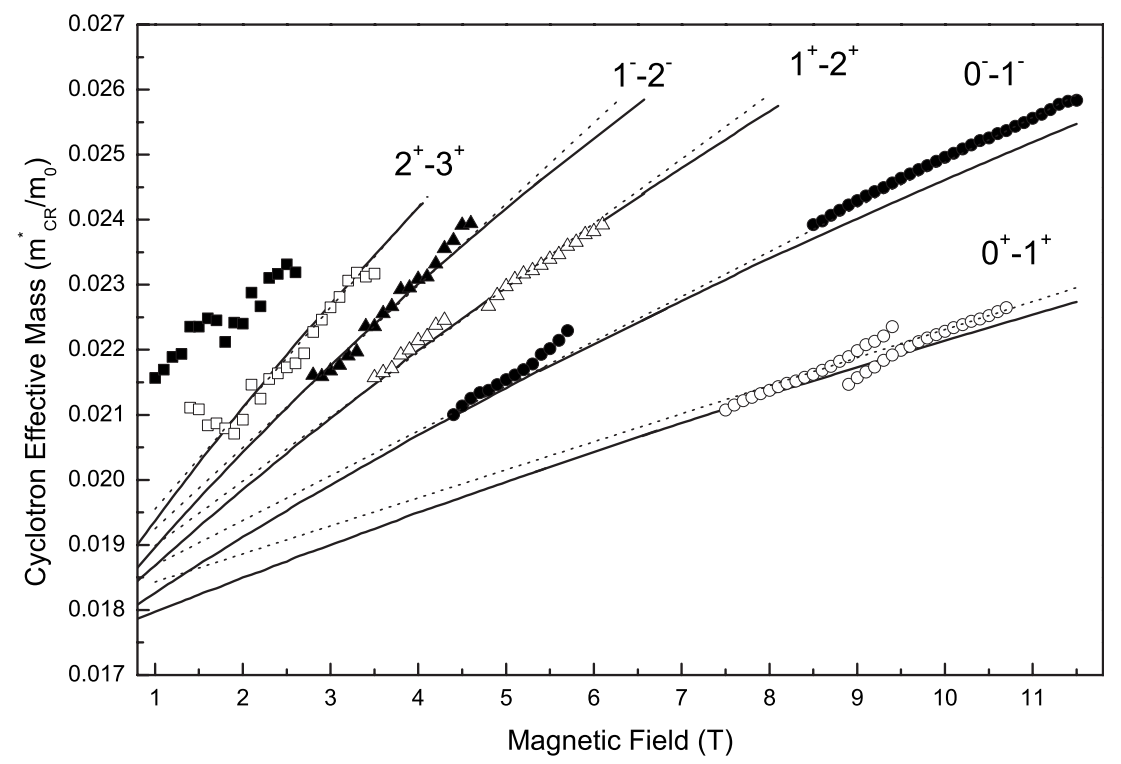

FIG. 3. Cyclotron mass deduced from spin-resolved cyclotron resonance energies as a function of magnetic field. The data points in the RMPC region have been removed for clarity. The calculated field-dependent cyclotron masses for each transition are shown as continuous lines and the linear cyclotron mass approximations are shown as dotted lines.

$\mathrm{T}$, where the $2^{+} \rightarrow 3^{+}$transition is resolved for approximately $7>\nu>4(2.3<B<4 \mathrm{~T})$, emerging from the unresolved transitions at lower fields. Spin-resolved CR is then present all the way through the data range, where it is predicted that the $0^{-} \rightarrow 1^{-}$transition will persist up to $\nu=1$ at $16.2 \mathrm{~T}$.

The cyclotron mass, $m_{\mathrm{CR}}^{* \pm}$, for each spin-resolved transition can be determined from the energies shown in Fig. 2 as $^{12}$

$$
m_{\mathrm{CR}}^{* \pm}=\frac{\hbar e B}{\left(E^{N+1, \pm}-E^{N, \pm}\right)} .
$$

This contains a field dependence that arises directly from the conduction band nonparabolicity. Figure 3 shows the measured cyclotron masses for each resolved transition (excluding for clarity those close to the polaron coupling). The cyclotron mass given by the $\mathbf{k} \cdot \mathbf{p}$ calculation is shown as a solid line and indicates a good fit with the data over the full range of magnetic fields for the five spin-resolved transitions.

As a simplification to the full eight-band $\mathbf{k} \cdot \mathbf{p}$ calculation the cyclotron mass may be given by the following expression in the range of fields considered here. To a first approximation $m_{\mathrm{CR}}^{*}$ can be considered linear with field so that,

$$
m_{\mathrm{CR}}^{*} \approx m_{0}\left(1+\alpha \frac{\epsilon}{E_{g}}\right),
$$

where $E_{g}$ is the bandgap $(0.24 \mathrm{eV}), m_{0}$ is the subband effective mass, and $\epsilon=\hbar^{2} k^{2} / 2 m_{0}$ is the uncorrected band-edge energy allowing the field dependence to be expressed as

$$
\epsilon=(N+1 \pm \Delta) \frac{\hbar e B}{m_{0}}
$$

for the transition $(N \rightarrow N+1)$. The Zeeman splitting of the Landau levels is accounted for by the term $\pm \Delta$. This approximation is shown as dotted lines in Fig. 3 for $m_{0}$ $=0.018, \alpha=1.16$, and $\Delta=0.23$ and achieves a good fit to the measured effective masses for all spin-resolved transitions in the ranges of magnetic field where they are active. Examination of the $\mathbf{k} \cdot \mathbf{p}$ calculation indicates that in fact the effective $g$ factor fits the linear expression $g^{*}=g_{0}+\beta E$, where $g_{0}$ is the band-edge $g$ factor, $\beta \approx 185 \mathrm{eV}^{-1}$, and $E$ is the energy above the band edge. This energy dependence can also be confirmed from the experimental data using the method described in Ref. 12.

\section{RESONANT SUBBAND LANDAU LEVEL COUPLING}

As demonstrated in Fig. 1 anticrossing behavior in the CR resonance energies as a function of magnetic field can be used to determine the subband coupling energy $E_{10}$. In Figs. 2 and 3 an anticrossing is clearly visible between 8.5 and 9.5 $\mathrm{T}$ in the transition $0^{+} \rightarrow 1^{+}$, at an energy just below $50 \mathrm{meV}$. This is clearly seen in Fig. 4(a), and while no such effect is discernible in the $0^{-} \rightarrow 1^{-}$branch, a slight broadening of the absorption peak can be seen. In order to demonstrate that this feature represents the resonant coupling between the ground and first excited subbands in the quantum well, the sample was remounted at an angle of $6^{\circ}$ to the magnetic field (TF configuration) and cyclotron resonance data, taken between 7.5 and $11.5 \mathrm{~T}$, are shown in Fig. 4(b).

The exact value of the RSLC is given by the point at which the split cyclotron resonance peaks of the upper and lower branches are of equal magnitude and linewidth. The cyclotron resonance peak positions are plotted in Fig. 5. In the TF configuration the data show signs of strong subband coupling, especially in the $0^{+} \rightarrow 1^{+}$resonance. As the field approaches $8.9 \mathrm{~T}$ the $0^{-} \rightarrow 1^{-}$peak also begins to widen and separate into two peaks, displaying characteristic anticrossing behavior. The anticrossings in both transitions are pinned at $47.9 \pm 1 \mathrm{meV}$. It is clear that the discontinuity observed in the PF configuration is a manifestation of the same effect and can be greatly enhanced by the deliberate introduction of a field component parallel to the 2DEG. We therefore identify this feature as RSLC between the two confined subbands in the InSb quantum well, giving $E_{10}=47.9 \pm 1 \mathrm{meV}$.

The presence of the significant anticrossing in the $0^{+}$ $\rightarrow 1^{+}$transition when the sample is in the PF configuration is noted. While a small, unintentional angular offset in the 


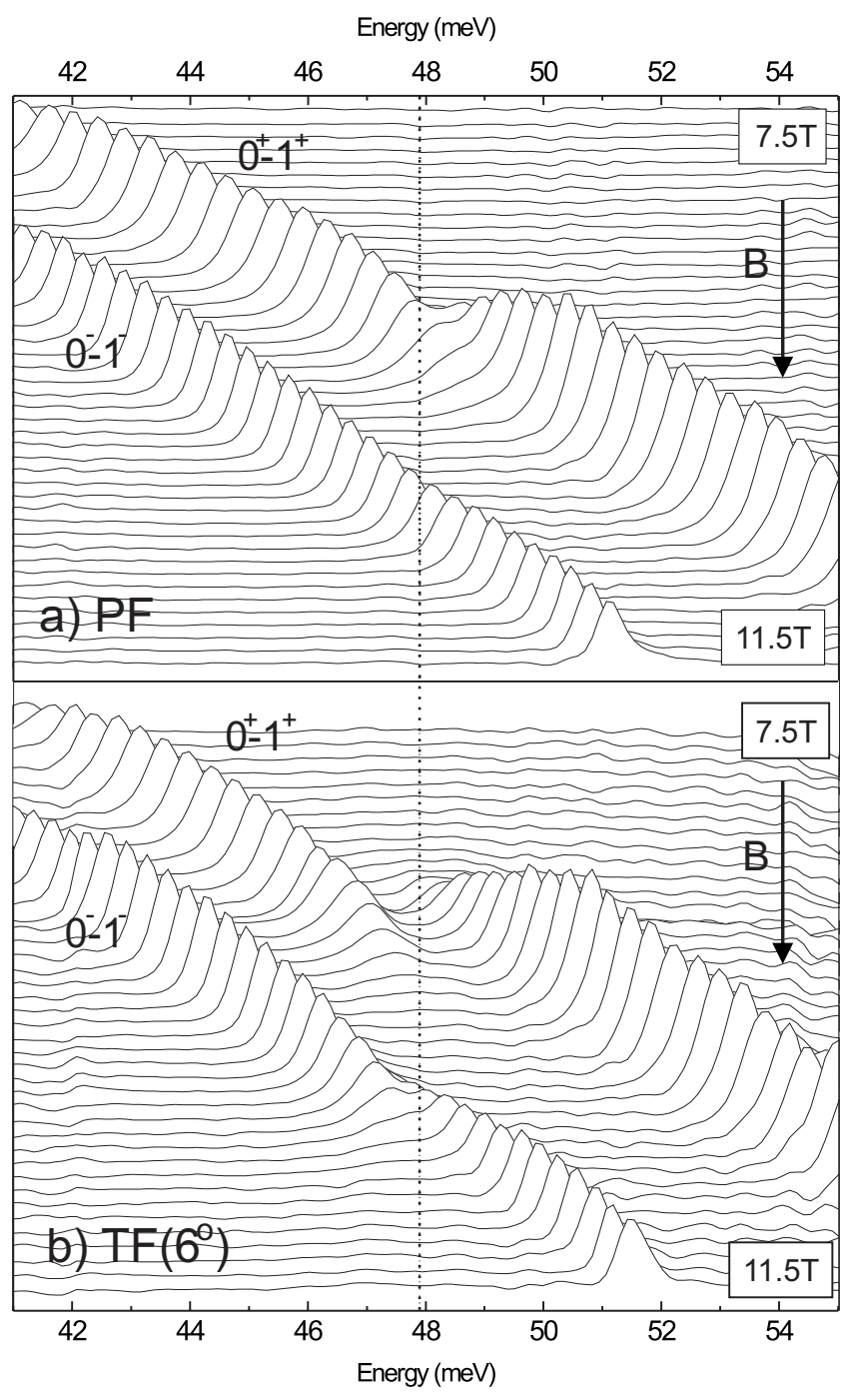

FIG. 4. Clyclotron resonance absorption spectra for the sample mounted in the (a) PF and (b) TF $6^{\circ}$ configurations for fields between 7.5 and $11.5 \mathrm{~T}$. Anticrossing is clearly visible in the $0^{+}$ $\rightarrow 1^{+}$resonance in the PF configuration, but much less so in the $0^{-} \rightarrow 1^{-}$resonance. In the TF configuration RSLC anticrossing is greatly enhanced in both transitions.

sample mounting is possible, it is unlikely to be sufficient to explain the magnitude of this feature.

According to the single-band approach, which treats the conduction and valance band wave functions separately, interaction between incident photons and quantum-well subband transitions is forbidden when there is no electric field component out of the 2DEG plane (PF configuration) $;^{13}$ furthermore the subbands are decoupled while the $2 \mathrm{DEG}$ is perpendicular to the field. However experimental evidence in GaAs/AlGaAs (Refs. 14 and 15) as well as InAs (Refs. 16 and 17) and InSb inversion layers ${ }^{17-19}$ indicates that weak intersubband coupling can take place even when the field lies entirely perpendicular to the 2DEG. Multiband $\mathbf{k} \cdot \mathbf{p}$ perturbation calculations have provided further theoretical evidence in favor of these results, showing the possibility for significant nonzero matrix elements for coupling between the ground and first excited states, in both symmetric and asym- metric quantum wells in the PF geometry. ${ }^{20,21}$ The existence of such coupling has important implications for normalincidence optical devices such as quantum-well infrared photodetectors (QWIPs) and intersubband lasers, where transitions between subbands by photons normal to the surface is desired. ${ }^{14,15}$ The origin of the breakdown in the $z(x, y)$ decoupling arises from the admixture of the $p$-type light and heavy hole states in the valance band and $s$-type conductionband states that are necessarily not considered in the singleband model. The transition probability for PF intersubband excitation is critically dependent on the strength of coupling between the conduction and valance bands, indicating that systems with strong nonparabolicity (such as InAs and InSb) will show greater evidence of this effect then wider bandgap materials such as GaAs. It is predicted that these transitions can be of the order of $1 \%$ of the strength of transitions induced by light fully polarized in the growth direction. ${ }^{21}$

Importantly, these considerations show that weak coupling between incident light in the PF configuration and intersubband transitions in the quantum well is not forbidden by selection rules, and will in fact be enhanced in the structures measured here because of their narrow bandgap. We believe that this coupling is consistent with the observation of nonzero intersubband coupling (RSLC) in these samples in the PF configuration.

\section{RESONANT MAGNETOPOLARON COUPLING}

Polaron coupling in polar semiconductors is a readily identifiable phenomenon in cyclotron resonance experiments by a characteristic anticrossing at the polaron energy. It was first observed in bulk materials, where the longitudinal optical (LO) phonon mode causes a macroscopic lattice distortion (and electric field) that couples to the electron motion (polaron). ${ }^{22}$ The excited electron will absorb and emit LO phonons as it moves through the lattice and a resonant interaction, known as RMPC, will be observed when the cyclotron resonance energy is equal to the LO phonon energy. This is analogous to making the coupling energy $\Delta E_{i}$ in Fig. 1 equal to $\hbar \omega_{\mathrm{LO}}$.

More recently similar effects have been reported in cyclotron resonance studies of GaAs quantum wells. ${ }^{23-25}$ Initially only a weak interaction was observed, and this was attributed to the PF geometry and the high carrier concentration of the sample $\left(1.28 \times 10^{12} \mathrm{~cm}^{-2}\right)$ screening the polaronic interaction. ${ }^{26,27}$ Further studies however have revealed that the RMPC can be enhanced by using a large tilt angle and lower carrier concentrations. ${ }^{24,25}$ It was also noted that within the quantum well the LO phonon is no longer a normal mode, as it couples to two-dimensional (2D) intersubband plasma oscillations (plasmons) in the 2DEG. Consequently, the RMPC occurs around a hybrid mode that consists of a coupled intersubband plasmon-LO phonon mode. ${ }^{24,28}$ The phonon coupling for 2DEGs in both PF and TF orientations has been treated by considering the dielectric tensor for a multilayer system, incorporating plasmonic excitations, and is found to be consistent with quantum mechanical treatments. ${ }^{29}$

A strong resonant coupling effect is identified in Fig. 2 close to the TO phonon obscured region and is attributed to 


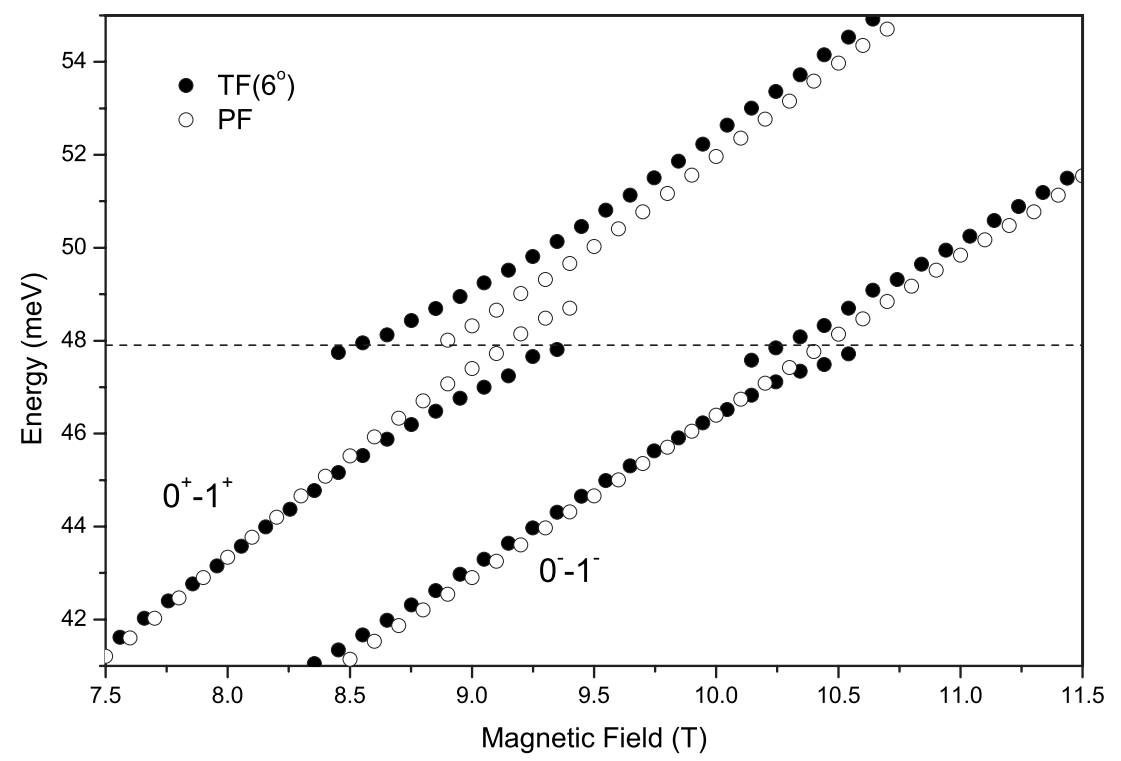

FIG. 5. Cyclotron resonance peak position as a function of magnetic field $B$. The presence of notable anticrossing in the $0^{+}$ $\rightarrow 1^{+}$transition while perpendicular to the field implies coupling of the $z$ and $(x, y)$ components of the electron wave function even for perpendicular field.

RMPC. Figure 6 shows the cyclotron resonance absorption spectra in the PF configuration covering this region (from 3.9 to $5.2 \mathrm{~T}$ ), and reveals a complicated pattern of absorption features labeled as A-F. By comparison with Fig. 2, B is identified as the $1^{-} \rightarrow 2^{-}$transition, and as predicted this transition decays as it approaches $\nu=3$. Feature A corresponds to $1^{+} \rightarrow 2^{+}$and persists throughout the TO phonon region where it splits into features labeled as $\mathrm{E}$ and $\mathrm{F}$. $\mathrm{E}$ and $\mathrm{F}$ are the lower and upper branches of the RMPC anticrossing. Similar anticrossing behavior is observed in features $\mathrm{C}$ and $\mathrm{D}$, which correspond to the $0^{-} \rightarrow 1^{-}$transition. This transition only occurs in the range $4>\nu>1(4.1<B<16.2 \mathrm{~T})$ and is not properly resolved until it passes though the hatched region. It

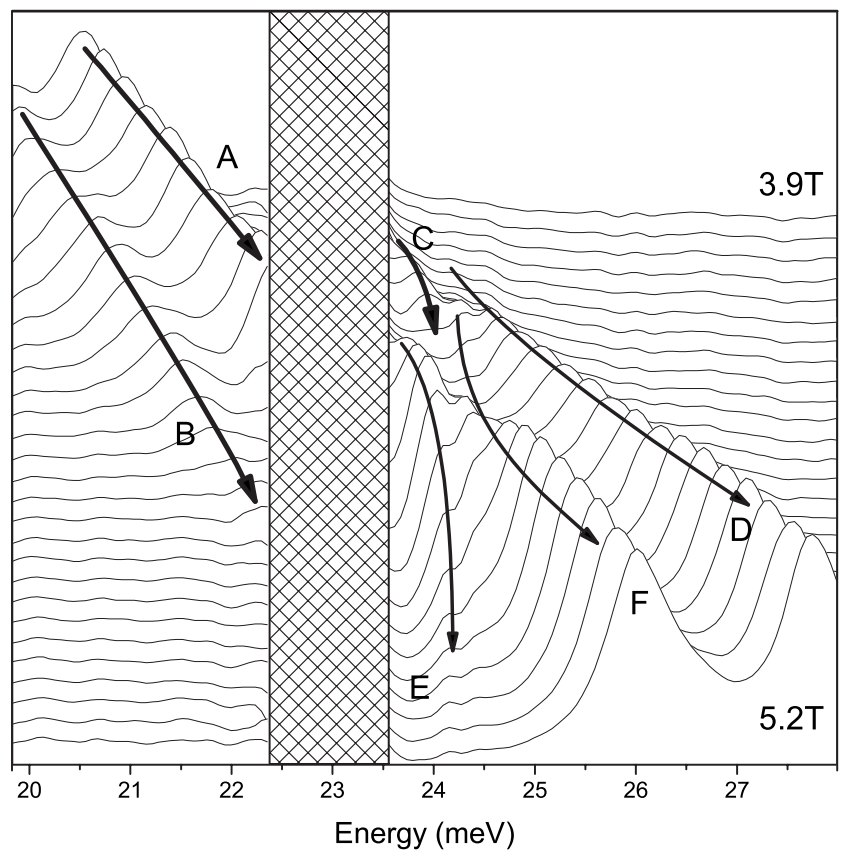

FIG. 6. Cyclotron resonance absorption spectra between 3.9 and $5.2 \mathrm{~T}$ in the region of the InSb TO and LO phonons. A significant range of energies are obscured by the TO phonon interaction. Absorption features A-F are identified. has not been possible to analyze the CR data close to the TO phonon energy due to poor signal to noise. Furthermore the $1^{-} \rightarrow 2^{-}$transition decays rapidly and the $0^{-} \rightarrow 1^{-}$transition only starts to become active in this region making precise analysis difficult. For the moderate doping levels examined here it might be expected that some interactions close to the TO phonon level would also be evident, ${ }^{27}$ but this has not been observed.

Figure 7 shows the CR energies as a function of magnetic field for the range shown in Fig. 6. It appears that both CR transitions that emerge from the obscured region couple strongly to an energy level just below the InSb-LO phonon

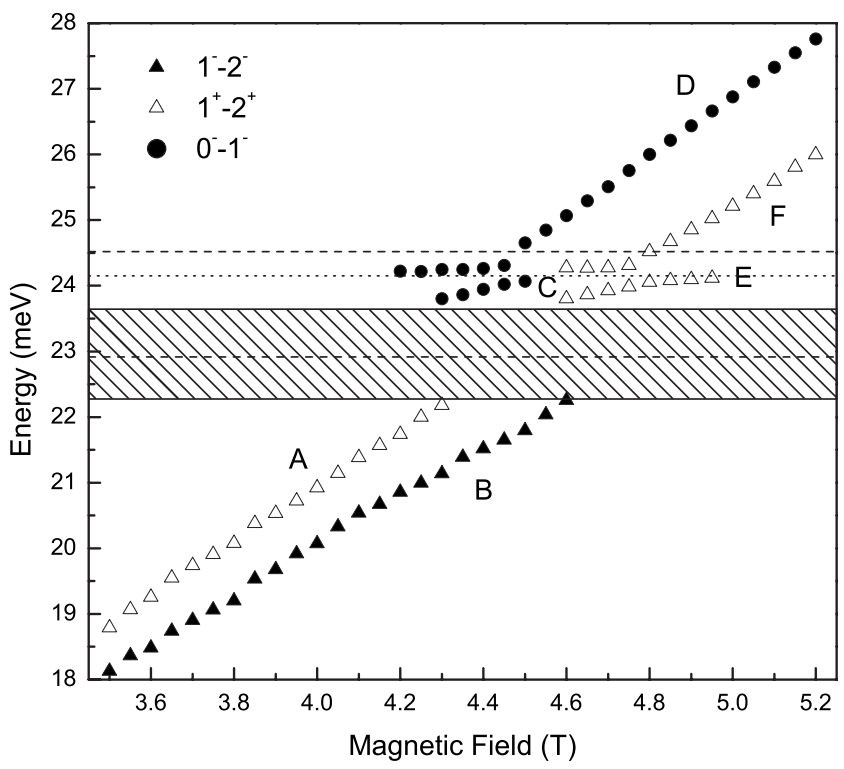

FIG. 7. Cyclotron resonance energy positions for transitions around the InSb phonon region for measurements taken in the PF configuration. Data are partly obscured around the InSb TO phonon energy. Strong anticrossing is seen in the $1^{+} \rightarrow 2^{+}$and $0^{-} \rightarrow 1^{-}$transitions. The InSb-LO phonon is shown as a dashed line. The calculated hybrid intersubband plasmon-LO phonon level is indicated by a dotted line at $24.15 \mathrm{meV}$. 
energy (shown as a dashed line), indicating that a hybrid mode exists in the 2DEG that is slightly modified from the bulk LO phonon energy.

While no coupling has been observed in GaAs structures in the PF configuration, the increased band mixing in narrow gap InSb has been shown to allow measurable coupling between intersubband transitions and light polarized in the 2DEG plane. It follows that, unlike in GaAs, the parallel and perpendicular motions are not strongly decoupled, and consequently intersubband plasmons may couple to the CR. With this in mind the RMPC observed in this sample will be the result of coupling between the CR and a hybrid intersubband plasmon-LO phonon mode. Following the approach taken by Faugeras et al..$^{24,25}$ for tilted GaAs samples the dielectric tensor component for perpendicular excitation is given by

$$
\epsilon_{z z}=\epsilon_{\infty} \frac{\omega^{2}-\omega_{\mathrm{LO}}^{2}}{\omega^{2}-\omega_{\mathrm{TO}}^{2}}+\frac{\omega_{p 2 \mathrm{D}}^{2}}{\omega_{10}^{2}-\omega^{2}}=0,
$$

where $\omega_{10}=E_{10} / \hbar$ has been found by examining the RSLC to be $47.9 \mathrm{meV}, \omega_{\mathrm{LO}}$ is the InSb-LO phonon energy, $\epsilon_{\infty}$ is the high-frequency dielectric constant, and $\omega_{p 2 \mathrm{D}}$ is the $2 \mathrm{DEG}$ plasma frequency, for the quantum-well width, $L$,

$$
\omega_{p 2 \mathrm{D}}=\sqrt{\frac{4 \pi e^{2} n_{s}}{L m^{*}}} .
$$

The energy of this hybrid intersubband plasmon-LO phonon mode is the solution to Eq. (4) and is depicted as a dotted line in Fig. 7 at $24.15 \mathrm{meV}$. This is close to the measured pinning value at which both spin-split transitions are seen to anticross in Figs. 6 and 7, implying that the CR couples to a mode that is very close to the LO phonon energy and that any correction due to the intersubband plasmon is small. Measurements undertaken on lower carrier concentration samples show similar effects, in particular, RMPC is observed with coupling slightly closer to the LO phonon energy, at a value consistent with the above interpretation.

\section{v. CONCLUSIONS}

We have observed spin-resolved cyclotron resonance in $\mathrm{InSb} / \mathrm{AlInSb}$ quantum-well heterostructures for magnetic fields greater than $2.2 \mathrm{~T}$. The strong nonparabolicity of the InSb heterostructure system allows significant $\Delta g$ splitting, which in turn allows resolution of spin-split Landau level transitions down to low fields. The resonant transitions have been identified by the range of filling factors for which they are observed, and by a close fit with a suitable eight-band $\mathbf{k} \cdot \mathbf{p}$ model. The accuracy of the fit is in further evidence by examination of the cyclotron mass where the characteristic mass of the resonant electrons corresponds well with values derived from the calculations.

Two regions of anticrossing are identified in the data and are attributed to resonant coupling effects. Between 8.5 and 9.5 $\mathrm{T}$ the $0^{+} \rightarrow 1^{+}$resonance splits in two with a constant pinning energy. This splitting can be increased by applying a small tilt angle to the sample with respect to the field, which also induces a similar splitting in the $0^{-} \rightarrow 1^{-}$transition. This is identified as the resonant subband Landau level coupling, giving $E_{10}=47.9 \pm 1 \mathrm{meV}$. The anticrossing in the PF configuration is found to be stronger than expected for an accidental angular misalignment and is attributed to conduction band-valance band mixing which is known to be significant in narrow bandgap structures such as these.

Another anticrossing occurs between 3.5 and $5.5 \mathrm{~T}$ with pinning just below the InSb-LO phonon energy, this is attributed to coupling between the cyclotron resonance and the hybrid intersubband plasmon-LO phonon mode in the quantum well. Observation of polaronic coupling just below the bulk LO phonon energy is consistent with similar measurements in GaAs-based systems ${ }^{24,25}$ with large tilt angles. The observation of RMPC in the PF configuration is facilitated by the band mixing identified in the RSLC measurements.

\section{ACKNOWLEDGMENTS}

Part of this work was supported by EuroMagNET under the EU under Contract No. RII3-CT-2004-506239 of the 6th Framework "Structuring the European Research Area, Research Infrastructures Action." *j.orr1@physics.ox.ac.uk

${ }^{1}$ T. Ashley, A. B. Dean, C. T. Elliott, G. J. Pryce, A. D. Johnson, and H. Willis, Appl. Phys. Lett. 66, 481 (1995).

${ }^{2}$ A. M. Song, A. Lorke, A. Kriele, J. P. Kotthaus, W. Wegscheider, and M. Bichler, Phys. Rev. Lett. 80, 3831 (1998).

${ }^{3}$ S. A. Solin, D. R. Hines, A. C. H. Rowe, J. S. Tsai, Y. A. Pashkin, S. J. Chung, N. Goel, and M. B. Santos, Appl. Phys Lett. 80, 4012 (2002).

${ }^{4}$ G. A. Khodaparast, R. C. Meyer, X. H. Zhang, T. Kasturiarachchi, R. E. Doezema, S. J. Chung, N. Goel, M. B. Santos, and Y. J. Wang, Physica E 20, 386 (2004).

${ }^{5}$ A. M. Gilbertson, M. Fearn, J. H. Jefferson, B. N. Murdin, P. D. Buckle, and L. F. Cohen, Phys. Rev. B 77, 165335 (2008).

${ }^{6}$ R. Merlin, Solid State Commun. 64, 99 (1987).
${ }^{7}$ M. A. Brummell, M. A. Hopkins, R. J. Nicholas, J. C. Portal, K. Y. Cheng, and A. Y. Cho, J. Phys. C 19, L107 (1986).

${ }^{8}$ J. G. Michels, R. J. Nicholas, G. M. Summers, D. M. Symons, C. T. Foxon, and J. J. Harris, Phys. Rev. B 52, 2688 (1995).

${ }^{9}$ J. M. S. Orr, A. M. Gilbertson, M. Fearn, O. W. Croad, C. J. Storey, L. Buckle, M. T. Emeny, P. D. Buckle, and T. Ashley, Phys. Rev. B 77, 165334 (2008).

${ }^{10}$ B. Nedniyom and R. J. Nicholas, Phys. Rev. B (to be published).

${ }^{11}$ M. J. Yang, R. J. Wagner, B. V. Shanabrook, J. R. Waterman, and W. J. Moore, Phys. Rev. B 47, 6807 (1993).

${ }^{12}$ M. J. Yang et al., Surf. Sci. 305, 271 (1994).

${ }^{13}$ L. C. West and S. J. Eglash, Appl. Phys. Lett. 46, 1156 (1985).

${ }^{14}$ Z. Yuan, Z. Chen, D. Cui, J. Ma, Q. Hu, J. Zhou, and Y. Zhou, Appl. Phys. Lett. 67, 930 (1995). 
${ }^{15}$ H. C. Liu, M. Buchanan, and Z. R. Wasilewski, Appl. Phys. Lett. 72, 1682 (1998).

${ }^{16}$ H. Reisinger and F. Koch, Solid State Commun. 37, 429 (1981).

${ }^{17}$ K. Wiesinger, H. Reisinger, and F. Koch, Surf. Sci. 113, 102 (1982).

${ }^{18}$ W. Beinvogl and J. F. Koch, Solid State Commun. 24, 687 (1977).

${ }^{19}$ W. Beinvogl and J. F. Koch, Surf. Sci. 73, 547 (1978).

${ }^{20}$ R. Q. Yang, J. M. Xu, and M. Sweeny, Phys. Rev. B 50, 7474 (1994).

${ }^{21}$ R. J. Warburton, C. Gauer, A. Wixforth, J. P. Kotthaus, B. Brar, and H. Kroemer, Phys. Rev. B 53, 7903 (1996).

${ }^{22}$ D. H. Dickey, E. J. Johnson, and D. M. Larsen, Phys. Rev. Lett. 18, 599 (1967).

${ }^{23}$ A. J. L. Poulter, J. Zeman, D. K. Maude, M. Potemski, G. Mar- tinez, A. Riedel, R. Hey, and K. J. Friedland, Phys. Rev. Lett. 86, 336 (2001).

${ }^{24}$ C. Faugeras, G. Martinez, A. Riedel, R. Hey, and K. J. Friedland, Physica E 22, 586 (2004).

${ }^{25}$ C. Faugeras, G. Martinez, A. Riedel, R. Hey, K. J. Friedland, and Y. Bychkov, Phys. Rev. Lett. 92, 107403 (2004).

${ }^{26}$ B. Zhang, M. F. Manger, and E. Batke, Phys. Rev. Lett. 89, 039703 (2002).

${ }^{27}$ S. N. Klimin and J. T. Devreese, Phys. Rev. B 68, 245303 (2003).

${ }^{28}$ L. Wendler and R. Pechstedt, J. Phys.: Condens. Matter 2, 8881 (1990).

${ }^{29}$ Y. Bychkov, C. Faugeras, and G. Martinez, Phys. Rev. B 70, 085306 (2004). 